\title{
Implementation of the Geriatric Patient-Aligned Care Team Model in the Veterans Health Administration (VA)
}

\author{
Jennifer L. Sullivan, PhD, Rina Eisenstein, MD, Thomas Price, MD, \\ Samantha Solimeo, PhD, MPH, and Kenneth Shay, DDS, MS
}

Background: Here, we describe the implementation of a specialty primary care medical home (PCMH) model called Geriatric Patient-Aligned Care Teams (GeriPACT) in the Veterans' Health Administration (VA) that is focused on serving older complex patients. In particular, our aims in this article are to describe how the GeriPACT model was developed and implemented in VA sites, provide a closer look at how GeriPACT functions by presenting a case study, and highlight data showing national variation in the implementation of GeriPACT staffing models and PCMH practices.

Methods: Stakeholder feedback regarding the GeriPACT model was obtained from a GeriPACT team and the director of GeriPACT in VA. Here, we present national data regarding variations in GeriPACT staffing and PCMH practices.

Results: Following the adoption and implementation of the GeriPACT model and release of the GeriPACT handbook, sites were able to adopt the model's principles. The VA's adoption of PCMH reinforced the mission of patient-centered primary care by integrating psychosocial and environmental determinants of health. This was accomplished with enhancements to staff support through new full-time employment equivalents, but also by optimizing staff productivity through improved team function and interpersonal care. The GeriPACT model was implemented in a bottom-up fashion that has led to variation in how GeriPACTs are structured and staffed, as well as how they conform to various PCMH principles.

Conclusions: GeriPACT is one approach for bringing an interdisciplinary, patient-centric perspective to primary care in a manner that can likely support the higher staffing costs with economies realized from diminished reliance on institutional placement and highly technologic health care. It is a model which can provide training for the next generation of providers and clinicians. ( $\mathrm{J}$ Am Board Fam Med 2018;31:456-465.)

Keywords: Implementation, Patient-Centered Medical Home, Primary Health Care, Geriatrics

Throughout the past decade, the patient-centered medical home $(\mathrm{PCMH})$ model has been widely adopted for the provision of primary care. This model uses team-based, comprehensive, accessible,

This article was externally peer reviewed.

Submitted 5 July 2017; revised 17 January 2018; accepted 20 January 2018.

From Center for Healthcare Organization and Implementation Research, VA Boston Healthcare System (JLS); ${ }^{2}$ Boston University School of Public Health, Boston, MA (JLS); Atlanta Veterans Affairs Medical Center, Atlanta, GA (RE, TP); Section of Geriatric Medicine and Gerontology, Emory University School of Medicine, Atlanta (RE, TP); Center for Comprehensive Access \& Delivery Research \& Evaluation Center and the VISN 23 Patient Aligned Care Team PACT Demonstration Laboratory, Iowa City VA Health Care System, Iowa City, IA (SS); Department of General Internal Medicine, University of Iowa Carver College of and coordinated care proposed to improve both quality and safety. ${ }^{1}$ Recent research suggests PCMH implementation has resulted in better quality and lower costs. ${ }^{2,3}$ Within a PCMH, primary

Medicine, Iowa City (SS); ${ }^{7}$ US Department of Veterans Affairs, Geriatrics and Extended Care Services, Washington, D.C. (KS).

Funding: This work was partially supported by VA QUERI Grant PEI-15-468 (Sullivan, PI). Dr. Solimeo receives support from the Center for Comprehensive Access \& Delivery Research and Evaluation, Department of Veterans Affairs, Iowa City VA Health Care System, Iowa City, IA (Award CIN 13-412), VA Health Services Research \& Development (Award CDA 13-272), and VISN 23 PACT Demonstration Lab, Iowa City VA Health Care System, Iowa City, IA, which is funded by the VA Office of Patient Care Services. 
care teams manage care for patient of all ages, focusing on preventative needs to chronic care management. However, older patients have unique needs that have likely not been incorporated into the current definitions of PCMH care provision. ${ }^{4}$ Population aging and the corresponding high prevalence of multiple chronic conditions ${ }^{5}$ means that typical PCMHs may struggle to provide the care coordination and longer appointment times those patients require.

To serve an older population comprehensively, PCMH will need to broaden the standard team to include social workers and pharmacists, as well as offer services to support aging in place. ${ }^{4}$ For example, older, frailer patients require comprehensive geriatric and behavioral health assessments, advanced care planning, and coordination of various home and community based services. Typical PCMHs may not possess knowledge of supportive services or the associated encounter time to assess patient needs. In the case of the most vulnerable older adults, a specialty PCMH with geriatrics expertise may be warranted. However, to date there is scant literature on the implementation of this type of model ${ }^{6}$ and little evidence of its effectiveness.

Within the Veterans Health Administration (VA), national efforts to implement both the PatientAligned Care Team (PACT) (eg, VA's version of the $\mathrm{PCMH}$ ) and a specialized Geriatric PACT (GeriPACT) model are maturing. GeriPACT provides frail, elderly patients and their caregivers access to the most appropriate care for their health care needs through a single point-of-contact for

Conflict of interest: none declared.

Corresponding author: Jennifer L Sullivan, $\mathrm{PhD}$, Center for Healthcare Organization and Implementation Research, VA Boston Healthcare System, 150 S. Huntington Ave (152M), Boston, MA 02131 (E-mail: jennifer.sullivan@va.gov).

Conflicting and Competing Interests: None identified.

Ethical Consideration: VHA Handbook 105805 (Veterans Health Administration 2011) provides guidance about authorization of manuscripts that have been developed through non-research activities (ie, without IRB approval under the authority of VHA operations). All VHA authors of this manuscript attest that the activities that resulted in producing this manuscript were not conducted as part of a research project, but as part of the nonresearch evaluation conducted under the authority of the Office of Geriatrics and Extended Care. The status of this work as quality improvement and not research was also confirmed following review by the Research and Development Committee at the VA Boston Healthcare System.

Previous dissemination: National survey data were presented at a 2017 Annual Academyhealth meeting poster session (New Orleans, LA). geriatric primary care. The premise is that the GeriPACT model, leveraging a multidisciplinary team and longer encounters, can do the following: (1) enhance coordination of biopsychosocial care, (2) provide high quality, personalized, proactive, and patient-centered care, and (3) promote a positive culture of service through reflexive team behaviors, processes, and systems. ${ }^{7}$ The GeriPACT model is hypothesized to improve patient and staff satisfaction and care quality, potentially reducing the likelihood of costly health care services, such as hospitalization or long-term institutionalization, as well as functional decline.

Given the lack of literature on specialty PCMHs, our aims in this article are the following: (1) to describe how the GeriPACT model was developed and implemented in VA, (2) to provide a closer look at how GeriPACT functions by presenting an example from the bronze team at the Atlanta VA Medical Center, and (3) to highlight national variation in the implementation of GeriPACT staffing models and PACT practices.

\section{Development and Implementation of VA GeriPACT}

A 1984 publication of results from a randomized control trial of inpatient comprehensive geriatric assessment and management at a VA site ${ }^{8}$ and the then-limited availability of VA primary care services illustrated that there were two factors contributing to the adoption of national VA programs for geriatric evaluation and management (GEM). However, patients benefitting from the comprehensive geriatric assessments interdisciplinary care planning and management often and precipitously declined without close monitoring once back in the community. To provide such monitoring, GEM continuity clinics were independently implemented at multiple VA sites through a program of multidisciplinary, team-based, veteran-centered, coordinated care, known as "Geriatric Primary Care" (GPC). ${ }^{9}$ The programs were of sufficient number (over 60 by 2007) and penetration (over 66,000 patients received care in GPC that same year) to warrant the issuance of a handbook in 2008.

In 2010, as the VA Office of Patient Care Services initiated national PACT implementation, GPC, with very little modification to actual practice, was rebranded as GeriPACT. By fiscal year 2015, about 60,000 patients were receiving GeriPACT services out of the 1.5 million enrolled veterans aged 65 and over, with a Jen Frailty Index 
of 3 or greater. A GeriPACT handbook, largely unchanged from its GPC predecessor, was issued to the field in 2015. ${ }^{7}$ However, GeriPACTs are not simply PACTs for older adults; the GeriPACT handbook specifies program goals, inclusion and exclusion criteria, and anticipated benefits to the hosting health care system.

Three characteristics differentiate GeriPACT from PACT - team composition, panel size, and provider experience. The core team for PACT consists of the provider and, for each full-time equivalent of a provider, 1 nurse care manager (RN), 1 clinical associate (customarily a Licensed Practical Nurse [LPN]), and 1 administrative associate (ie, Medical Support Assistant [MSA]) dedicated to the team. ${ }^{10}$ The day-to-day role of nurses within PACTs differs by team, but in general, the $\mathrm{RN}$ is responsible for chronic disease management, protocol-guided medication adjustments, telephone care, and direct patient care, whereas the clinical associate administers routine vaccinations, records vitals, provides patient education, and prepares patients for the encounter. ${ }^{11}$ An individual PACT shares a single mental health provider, clinical pharmacist, and other disciplines, such as nutrition or social work, with multiple teams. In contrast, a core GeriPACT includes typical PACT staff as well as social work and clinical pharmacist support. Social workers and clinical pharmacists review each patient's record in advance of appointments and are present in the clinic, participating in huddles and team meetings, and interacting with every patient. The social worker addresses family dynamics and assists in advanced care planning, coordinates VA and community-based services, and facilitates transitions between settings of care. The pharmacist undertakes medication reconciliation to reduce polypharmacy and addresses necessary adjustments with or independent of the provider and undertakes patient and caregiver medication education.

In PACT, panel size is typically capped at 1200 patients. In a GeriPACT, panels are limited to two-thirds the size of a PACT panel (averaging 800 per full-time provider). Finally, whereas PACT providers treat patients of all ages, including caring for frail elderly without specialized training to do so, GeriPACT providers have received either advanced training in geriatric medicine (board certified or eligible) or demonstrate advanced experience in geriatric care, as outlined in the VHA handbook ${ }^{7}$.

\section{GeriPACT Implementation at the Atlanta VA Medical Center}

The Bronze Outpatient Clinic was established as a dedicated geriatric medicine clinic in 1999 and transitioned to the medical home model (GeriPACT) in 2011. Patients referred to the Bronze GeriPACT from the regular PACT have either demonstrated one or more geriatric syndromes, or are age 80 or older. As of December 2017, there are 1493 patients enrolled in Bronze GeriPACT, with an average age of 84 years. The GeriPACT uses geriatric principles, such as preferences for treatment, goals of care, evaluation and preservation of function, and psychosocial determinants of health. Treatment and screening choices are based on ageappropriate guidelines and shared decision making with the patient and/or caregivers. The bronze team also coordinates care with subspecialty care and has oversight of care transitions after hospital discharge, with the goal of supporting aging in place and reducing institutional care use. Bronze team members get to know their patients and the patients know their team, which saves a great deal of time and effort, leading to better quality of care for the patient and improved personal and professional satisfaction of the staff. The team environment is collegial and family-like, with good working relationships among staff and open communication to help provide patients the best care possible.

In addition to informal team communication throughout the day, the Bronze GeriPACT uses daily team huddles. The preparation for a visit starts with a huddle the day before, with the physician, RN care manager, and LPN meeting to discuss the following day's patients. Patient charts are reviewed for any recent changes in medications, specialty care encounters, hospitalizations, lab results, or missing vaccinations. This review enables the LPN to notify the patient of lab work, logs, or other information required for their visit during their reminder call. The huddle enhances care coordination, as the team can help the patient manage multiple appointments on the same day with different members of the care team or other subspecialty clinics. Huddles are valued by team members as they feel better prepared to anticipate the needs of the patients seen in the clinic the next day.

Bronze GeriPACT strongly adheres to the "open access" model mandated by PACT design. Patients are seen on walk-in, same-day, and prescheduled bases. Every effort is made to ensure that 
continuity of care with the patient's primary care physician is preserved. Walk-in patients are almost always seen by their primary providers. Continuity with the same provider is also enhanced by offering telephone-based care during regular clinic hours, communicating via a secure messaging platform. GeriPACT also participates in an after-hours live telephone assistance program staffed by clinic nurses. These nurses provide advice and potentially reduce emergency department use when the clinic is closed. As a result, Bronze GeriPACT matches or exceeds key performance metrics of the general PACT teams at the medical center. For example, Press Ganey provider satisfaction scores are between $7 \%$ and $23 \%$ higher for GeriPACT providers than comparable PACT providers. Although GeriPACT patients are older and sicker than PACT patients, emergency department use in GeriPACT is comparable to that in PACT.

Although Bronze GeriPACT strives to never turn away elderly patients who seek care, the Bronze GeriPACT has also faced challenges in care provision. Our current staff have limited support from clerical staff, social workers, and the mental health provider. A major limitation to care efficiency has been space limitation (ie, one examination room dedicated per provider), which is expected to be remedied in a future clinic redesign. Despite implementing a cohesive, collaborative team dedicated to geriatrics, Bronze GeriPACT shares challenges reflective of national variation in PACT and GeriPACT implementation. ${ }^{12,13}$

\section{National Variation in GeriPACT Implementation}

To assess the state of GeriPACT implementation, through variations in team structures and practice, we developed and administered a national web-based survey of GeriPACTs. Within the survey, GeriPACT structural components items included questions about staff mix and ratios, and GeriPACT practices items included domains found in the American College of Physicians Medical Home Builder Survey, such as access, scheduling, care coordination and transitions of care, population management, quality and performance improvement, and specific care process (eg, addressing barriers to communication, use of self-management tools, and involvement of patients in care).

\section{Methods}

With support from the VA Office of Geriatrics and Extended Care, we identified 71 VA health care systems nationwide with GeriPACT programs. In July 2016, we contacted the physician leaders by e-mail, providing them with a survey link. The survey was in the field for 4 weeks, during which time we sent two follow-up e-mails requesting participation. In addition, the National Director of VA Geriatric Programs (KS) sent an e-mail encouraging sites to take part in the study. Forty-four of the 71 facilities with a GeriPACT participated in the survey, resulting in a response rate of $62 \%$. Data analysis was conducted in SAS Version 9.3 (SAS Institute Inc, Cary, NC). We used descriptive statistics, including frequencies to examine team composition and PACT components and means to assess staff full-time employment equivalents (FTEE). Eight GeriPACTs declined to answer questions regarding PACT practices. We found no systematic differences (eg, facility size, GeriPACT staffing, or team composition) between sites who provided PACT principles information and those who refused.

\section{Results}

\section{Implementation of GeriPACT Staffing Elements}

Within the 44 responding sites, there were 102 GeriPACTs; most sites had multiple GeriPACTs. As expected, many GeriPACTs considered the core team to include a social worker, $\mathrm{RN}$, physician/ geriatrician, MSA, LPN, and a clinical pharmacist. See Table 1 for a summary of team composition. It is notable that many GeriPACTs (89\%) reported lacking expected core team members, including a pharmacist, LPN, or MSA. Positively, GeriPACTs reported engagement with many other disciplines, such as dieticians, psychologists, and physical therapists.

An analysis of FTEE assignments reported in Table 2, reveals that despite many GeriPACTs having critical staff in place, they were not always appointed at sufficient effort. It is important to note that of the core team members listed in the GeriPACT handbook, only 3 were staffed with mean FTEE coverage at or above 0.7 FTEE, namely the physician/geriatrician, LPN, and RN. The mean FTEE for the remaining core team members, social worker, MSA, and pharmacist, ranged between 0.35 and 0.56 . The extended team 
Table 1. Team Member Composition $(N=44)$

\begin{tabular}{lc}
\hline Team Role: Core & $\mathrm{N}$ \\
\hline Social worker & 37 \\
Registered nurse & 33 \\
Physician/geriatrician & 32 \\
Clerical associate & 25 \\
Licensed practical nurse & 24 \\
Pharmacist/clinical pharmacist specialist & 23 \\
\hline Team Role: Extended & $\mathrm{N}$ \\
\hline Dietician & 14 \\
Psychologist/geropsychologist & 14 \\
Nurse practitioner & 12 \\
Other unspecified & 7 \\
Speech or language pathologist & 4 \\
Clinical registered nurse specialist & 3 \\
Physician assistant & 2 \\
Health administrator & 2 \\
Occupational therapist & 2 \\
Physical therapist & 2 \\
Nurse aide & 1 \\
Chaplain & 1 \\
\hline
\end{tabular}

members had a low mean FTEE coverage in a range from 0.20 to 0.38 .

Implementation of PACT Practices within GeriPACTs As anticipated, implementation of PACT practices within GeriPACTs varied. In Table 3, we summa- rize those practices most commonly implemented and also provide the average number of practices implemented by domain. In contrast, in Table 4, we summarize the PACT principles least often implemented by GeriPACTs. As a way of succinctly presenting our results, we only detail PACT practices when the majority of GeriPACTs report using them (ie, 75th percentile).

A majority of teams had implemented access and scheduling practices, such as providing non-faceto-face clinical guidance to patients, scheduling each patient with their regular provider except when the clinician is unavailable, and providing same-day appointments. Less commonly implemented were related practices of scheduling group visits and scheduling dedicated phone hours. A majority of GeriPACTs used 16 of the 22 total care coordination and transitions practices. Of these, the most frequently implemented included reviewing information received from other facilities to identify what follow-up support a patient needs, assessing barriers when patients do not move toward their treatment goals, and reviewing charts in advance of visits to anticipate patient needs (ie, as part of the huddle). Interestingly, few GeriPACTs had implemented transition of care practices for establishing processes and expectations for notification of admissions to community hospitals or

Table 2. Distribution of Geriatric Patient-Aligned Care Team Member Full-Time Employment Equivalent

\begin{tabular}{|c|c|c|c|c|c|}
\hline Team Role: Core & Mean FTEE & SD & Min & Max & $\mathrm{N}$ \\
\hline Physician/geriatrician & 0.73 & 0.39 & 0.08 & 1.50 & 33 \\
\hline Registered nurse & 0.71 & 0.33 & 0.07 & 1.00 & 29 \\
\hline Licensed practical nurse & 0.75 & 0.45 & 0.08 & 2.00 & 19 \\
\hline Clerical associate & 0.56 & 0.34 & 0 & 1.00 & 23 \\
\hline Social worker & 0.43 & 0.35 & 0 & 1.00 & 32 \\
\hline Pharmacist/clinical pharmacist specialist & 0.35 & 0.32 & 0.01 & 1.00 & 26 \\
\hline Team Role: Extended & Mean FTEE & SD & Min & Max & $\mathrm{N}$ \\
\hline Nurse aide & 1.00 & - & 1.00 & 1.00 & 1 \\
\hline Nurse practitioner & 0.77 & 0.48 & 0 & 2.00 & 17 \\
\hline Clinical resident nurse Specialist & 0.59 & 0.37 & 0.30 & 1.00 & 3 \\
\hline Physician assistant & 0.50 & 0.41 & 0 & 1.00 & 4 \\
\hline Occupational therapist & 0.38 & 0.48 & 0 & 1.00 & 4 \\
\hline Physical therapist & 0.38 & 0.48 & 0 & 1.00 & 3 \\
\hline Health administrator & 0.31 & 0.09 & 0.25 & 0.38 & 2 \\
\hline Psychologist/geropsychologist & 0.26 & 0.29 & 0 & 1.00 & 12 \\
\hline Dietician & 0.24 & 0.30 & 0 & 1.00 & 9 \\
\hline Psychiatrist/geropsychiatrist & 0.20 & 0.26 & 0 & 1.00 & 12 \\
\hline Speech or language pathologist & 0.08 & 0.14 & 0 & 0.25 & 3 \\
\hline
\end{tabular}

FTEE, full-time employment equivalents; SD, standard deviation. 
Access and Scheduling (average practices implemented $=6.89 / 10$ )

provides non-face-to-face clinical guidance to patients

schedules each patient with a personal clinician except when the clinician is unavailable

provides a same-day appointment if clinically indicated, excluding emergency department

Care Coordination (average practices implemented $=17.9 / 22$ )

reviews information received from other facilities to identify what follow-up support a patient needs

assesses barriers when patients do not move toward their treatment goals (eg, medication adherence, costs of

care, and lack of family support).

incorporates external records into the practice chart

reviews charts in advance of visits to anticipate patient needs

Population Management (average practices implemented $=8.05 / 13$ )

incorporates evidence-based guidelines into everyday workflows for important conditions seen by Geriatric

Patient-Aligned Care Team

identifies patient on particular medications who need monitoring or evaluation and reminds them of this need

identifies patient who might benefit from additional coordination/care management services and contacts

them about these options

Care Processes (average practices implemented $=11.38 / 20$ )

involves patients in their own decision making

offers patients information about Advance Directives

routinely determined whether a patient has difficulty with hearing, vision, or other barriers to communication

provides a list of agencies, community-based organizations, or other entities to patients/families that support

patient self-management (when appropriate).

Quality Improvement (average practices implemented $=9.69 / 19$ )

measure or receive data on the performance of the practice on key clinical and administrative processes

Organizational Elements (average practices implemented $=9.36 / 14$ )

works as a team

notifies patients of all abnormal results

provided written case summary and transition plans for patients transferring care to another clinician or facility.

With regard to population management, many GeriPACTs used at least 4 population management practices. The 2 practices most often implemented were medication-focused monitoring and evaluation, and identifying patients who might benefit from additional care coordination/care management services to support patient self-management. Least frequently implemented practices included generating a list of empaneled patients who needed attention and incorporating guidance for clinical guidelines into flowsheets and standing orders to facilitate evidence-based medicine.

The most often reported care processes included shared decision making, offering patients information about advanced directives, and identifying communication barriers (eg, difficulty with hearing and vision). The least frequently implemented care practices included having a committee of patients to advise the facility, involving patients and families in developing information and education materials for GeriPACT, involving patients/families in facilitating programs and group activities, and connecting patients and families with similar medical conditions.

The majority of GeriPACTs had implemented a quality improvement practice of measuring or receiving data on the performance of the practice on key clinical and administrative processes, but less than two-thirds of GeriPACTs reported that they consistently monitored their performance. The least often implemented quality improvement practices were the following: monitoring turnaround times for lab tests, phone calls, and other servicerelated activities; collecting data on medication errors or other safety events; and reviewing data on patient confidence in their clinicians. Within the organization of care domain, over three-quarters of 


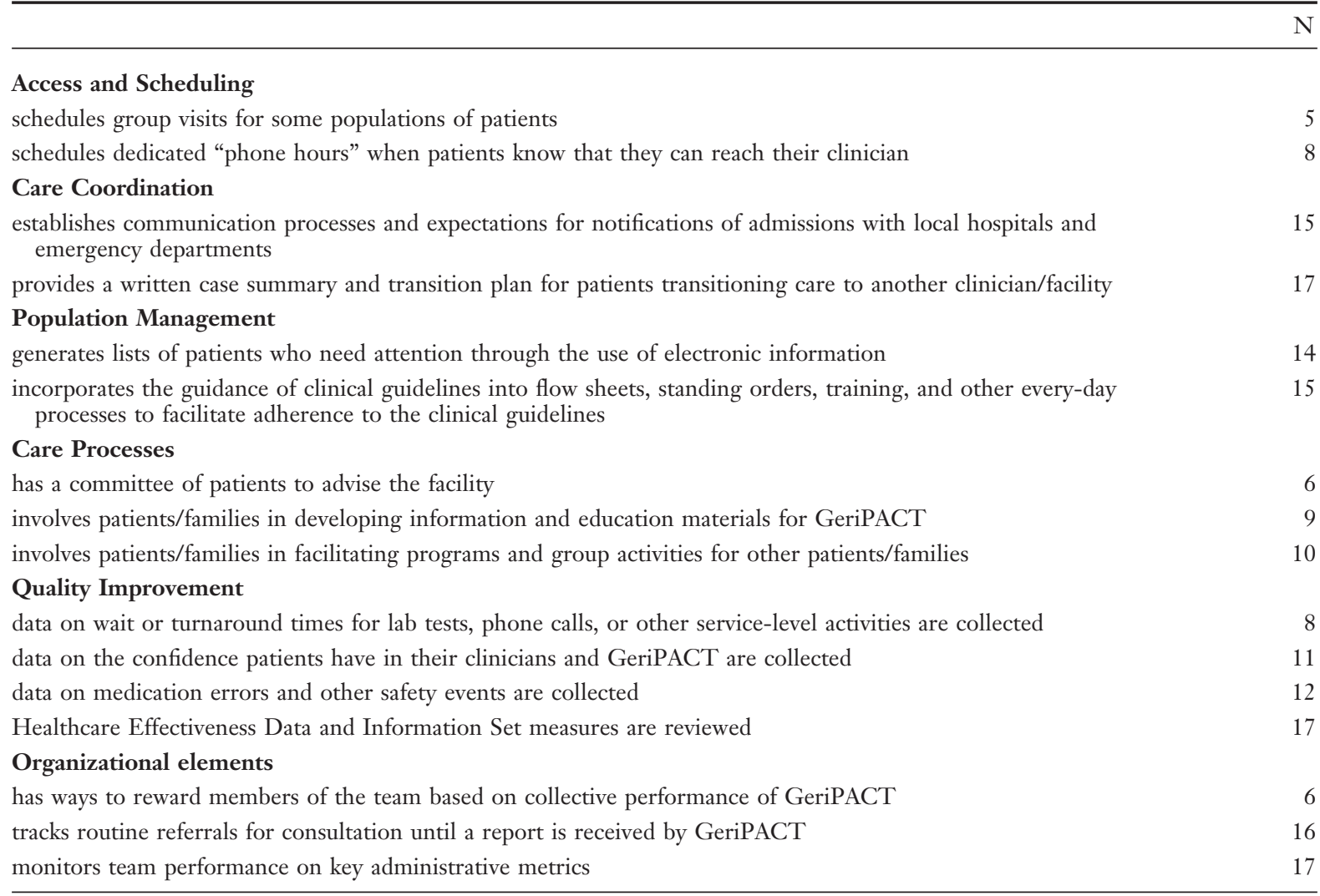

GeriPACT, Geriatric Patient-Aligned Care Team.

GeriPACTs implemented "working as a team." Least implemented practices included having ways to reward team members based on collective performance of GeriPACT and monitoring team performance on key administrative metrics.

In summary, we found that there was variation in both the way GeriPACT team staffing was structured and in the PACT practices implemented in the GeriPACT model. We discuss these findings in more depth in the discussion.

\section{Discussion}

The underlying principle of $\mathrm{PCMH}$ is that primary care should strive to be patient-centered-that is, focused on each patient's individual needs and characteristics, rather than compelling patients to adapt to the constraints posed by the health system. VA adoption of PCMH reinforces the mission of patient-centered primary care by integrating psychosocial and environmental determinants of health. Implementation was accomplished with enhancements to staff support through new FTEE, but also by optimizing staff productivity through improved team function and interpersonal care. The expansion of primary care efforts to include care coordination and patient and family education included the expectation that a broader range of patient needs would be thereby addressed.

Nationally, GeriPACT was developed in response to the existence of a small but complex subset of patients whose primary care needs were not able to be addressed adequately in the original primary care structure (eg, within the standard primary care 30-minute grid). Compounding the usual primary care time limitations, older patients require providers to review extensive medical records and to accommodate slower moving adults with ambulatory or cognitive impairments. But in many cases, time constraints were not the sole impediment, and clinicians with advanced training or other expertise in dealing with patients with complex medical conditions (eg, geriatricians) found not only their advice but also patient management 
contributions to be in high demand from their primary care colleagues.

GeriPACT implementation has progressed in the face of the new competencies and team care offered by PCMH. The number of sites offering GeriPACT has increased by almost $50 \%$ in 7 years. Furthermore, of the sites offering GeriPACT, more than half had 2 or more GeriPACT teams, suggesting organizational support and patient need. GeriPACTs' growth has been assisted by a national informal community of practice that has emerged; it is supported by a monthly, voluntary conference call and has provided the groundwork for a national 3-day GeriPACT summit in 2016. The community of practice fostered the identification of best practices and identified sites and personnel who can advise newer or struggling GeriPACTs.

The survey results also reflect the disseminated, rather than centrally-directed, origin of GeriPACT and demonstrate diversity in staffing and practices that mirror local needs and resources. For example, only about half of the GeriPACTs conform to the prescribed team staffing. These results are similar for the PACT model, where it proved difficult to achieve full staffing nationally ${ }^{14}$, despite PACT implementation being centrally-directed and resourced to a far more extensive degree than was GeriPACT. VHA leadership exerts considerable flexibility in how they staff their programs and distribute resources. This variation, combined with a national shortage of clinicians with advanced expertise in geriatrics, constrains GeriPACT staffing and the implementation of new teams. Clearly, GeriPACTs with a full complement of staff are better resourced to serve patients, both due to the additional FTEE and the wider range of professional skill sets. Yet, it is also likely that an understaffed, but geriatrics-trained GeriPACT team will possess the ability to provide a higher level of patient-centered care due to the interdisciplinary nature of geriatric medicine.

The survey results illustrate how GeriPACTs are providing services above and beyond standard PACT care. Most notable among these were practices that would be expected of programs devoted to preserving frail elders' function, autonomy, and ability to age in place. Included among these were the following: coordination of services; facilitating the continuity of care during transitions among different sites of care; assisting in advance-care planning and assessing and addressing barriers to fulfillment of care goals; and conducting medical record reviews in advance of appointments.

In turn, survey data show how standard PACT practices may at times be a poor fit for GeriPACTs and the populations they serve. The infrequent implementation of evidence-based population health practices in GeriPACTs may reflect the relative paucity of such recommendations applicable to frail, elderly populations. Similarly, GeriPACTs' infrequent implementation of calculated metrics designed for population management is likely attributable to empanelment and case mix in PACT versus GeriPACT. In keeping with their advanced age, medical complexity, and high use of health services, virtually all GeriPACT patients could be identified as "high risk" patients by using standard metrics. In contrast, a PACT panel is more apt to be comprised of a wider range of clinical presentations, ranging from younger, healthy patients in the workforce to the frail elderly. Thus, PACT providers are more likely to benefit from and use mechanisms for identifying the highest-risk patients.

Despite the limitation of these tools for GeriPACT, GeriPACTs can benefit from the establishment of performance metrics. Recent recognition of the need for such standards by providers and leadership has led to efforts to develop a GeriPACT dashboard populated by administratively derived data that will inform program managers at the team, medical center, regional level, and national level. Currently in development, the dashboard will consist of 4 domains, team structures, patient characteristics, risk factors/business case indicators, and processes of care. The dashboard will provide useful means by which national leadership can identify practice patterns, potentially pairing high-performing sites with lower-performing ones to foster continuous improvement, and individual GeriPACTs can leverage data to provide local justifications for initiating or expanding GeriPACT availability.

\section{Discussion}

The VA is a capitated health system that cares for a predominantly elderly male patient population. It is therefore reasonable to examine the lessons learned from GeriPACT in terms of their transferability to other health systems that likely care for age and gender distributions more reflective of the general population, as well as health care systems that have to be attentive to different reimbursement schemes. 
Under the traditional Medicare fee-for-service model, many GeriPACT services are not reimbursable due to their lack of a "face-to-face" component of care (eg, patient communication via electronic means or caregiver counseling). Some of these costs are indirectly covered in quality-based bonus payments by Medicare Advantage programs and, more recently, Medicare's Quality Payment Program. However, many professional organizations have pursued the establishment of current procedural terminology codes for care coordination and other tasks that do not occur with the direct presence of the patient. ${ }^{15,16}$ Although these may help defray the cost of care provided in this manner, they may not be sufficient for the hiring of persons to comprise a multidisciplinary team (eg, pharmacist and social worker).

Team-based care practices emblematic of GeriPACT can be implemented under the current payment structure. For example, daily huddles help prepare the care team for the day's work, yet only take several minutes to accomplish. Empowering nurses via standing orders to perform routine care such as immunizations and medication reconciliation helps meet quality measures under the Quality Payment Program and can result in increased reimbursement through the pay for performance model. In the accountable care model, GeriPACT principles can be deployed more broadly, as the reimbursement system favors the performance model over the service model. In those cases, cost savings through decreased use of higher levels of care may often cover the cost of members of the team that are not traditionally reimbursed under Medicare (such as pharmacy). For example, a national analysis of VA GeriPACT activity compared VA plus Medicare costs of patients managed 2012 to 2013 by GeriPACT to the costs incurred by a propensity-matched population of patients managed by PACT. VA costs were nearly $\$ 6000$ less per GeriPACT patient, and VA plus Medicare costs were over $\$ 6800$ less per GeriPACT patient $(P<.0001)$, strongly suggesting a reasonable likelihood for cost-effective applicability of this model to non-VA populations. ${ }^{17}$

There is little question that the increase in the number of elderly Americans will continue to pose a steadily growing demand for health care and supportive services. Much of the existing United States health care system is tightly wedded to a complex infrastructure and the medical expertise trained to leverage those resources to diagnose and treat disease. An uncertain but unquestionably growing proportion of the health needs of the aging population is better addressed through services that do not fit this traditional health care model. With increasing efforts devoted to discerning patient and family preferences about goals of care and quality of life, that proportion can be expected to continue to grow. GeriPACT is one approach for bringing an interdisciplinary, patient-centric perspective to primary care in a manner that can likely support the higher staffing costs resulting from diminished reliance on institutional placement and highly technologic health care. It is a model which can provide training for the next generation of providers and clinicians. Furthermore, it is reasonable to project that fee-for-service systems will likely take note of the cost savings that are being achieved by committing resources to case management, patient education, family involvement, and facilitated transitions in care and will adjust their reimbursement schedules to favor the wider adoption of such practices.

We acknowledge Ted Johnson, MD, for reviewing the manuscript. We also thank our project staff for assisting on the GeriPACT evaluation survey team, including Omonyêlé Adjognon, ScM, Kimberly Harvey, MS, Melissa J. Steffen, BS, MPH, Marlena Shin, JD, MPH, Jennifer Moye, PhD, ABPP, and Orna Intrator, $\mathrm{PhD}$.

The views expressed in this article are those of the authors and do not necessarily reflect the position or policy of the United States government or the Department of Veterans Affairs.

To see this article online, please go to: http://jabfm.org/content/ 31/3/456.full.

\section{References}

1. United States Department of Health and Human Services. Defining the PCMH. 2015; Available from: http://pcmh.ahrq.gov/page/defining-pcmh.

2. Nielsen M, Buelt L, Patel K, Nichols LM, Fund MM. The patient-centered medical home's impact on cost and quality. Milbank Memorial Fund. 2014.

3. Alexander JA, Bae D. Does the patient-centred medical home work? A critical synthesis of research on patient-centred medical homes and patient-related outcomes. Health Serv Manage Res 2012;25:51-9.

4. The Gerontologial Society of America. Patient-centered medical homes and the care of older adults. 2016. Available from: https://changeagents365.org/ resources/patient-centered-medical-home-network/ Roadmap_PCMH_Change\%20AGEnts.pdf. 
5. Lochner KA, Cox CS. Prevalence of multiple chronic conditions among Medicare beneficiaries, United States, 2010. Prev Chronic Dis 2013;10:E61.

6. Patel NK, Jaén CR, Stange KC, Miller WL, Crabtree BF, Nutting P. Patient centered medical home: A journey not a destination. In: Geriatrics models of care. Switzerland: Springer International Publishing;2015. p. 155-62.

7. Department of Veterans Affairs. Geriatric patientaligned care team (GeriPACT). In: VHA handbook 1140.07. Washington, D.C.: Department of Veterans Affairs. 2015. Available from: file://C:/Users/ rasgon_lab/Downloads/1140_07_

HK_2015_06-17_amended_2015-11-06\%20(2).pdf

8. Rubenstein LZ, Josephson KR, Wieland GD, English PA, Sayre JA, Kane RL. Effectiveness of a geriatric evaluation unit: A randomized clinical trial. N Engl J Med 1984;311:1664-70.

9. Shay K, Schectman G. Primary care for older veterans. Generations 2010;34:35-42.

10. Rosland AM, Nelson K, Sun H, et al. The patientcentered medical home in the Veterans Health Administration. Am J Manag Care. 2013;19:e263-272.

11. Stewart KR, Stewart GL, Lampman MM, Wakefield B, Rosenthal G, Solimeo SL. Implications of the patient centered medical home for nursing practice. J Nurs Adm 2015;45:569.

12. Farmer MM, Rose DE, Rubenstein LV, et al. Challenges facing primary Care practices aiming to implement patient-centered medical homes. J Gen Intern Med 2014;29:555-562.

13. Rodriguez HP, Giannitrapani KF, Stockdale S, Hamilton AB, Yano EM, Rubenstein LV. Teamlet structure and early experiences of medical home implementation for veterans. J Gen Intern Med 2014; 29:623-631.

14. Helfrich CD, Simonetti JA, Clinton WL, et al. The association of team-specific workload and staffing with odds of burnout among VA primary care team members. J Gen Intern Med 2017;32:760.

15. Merrell K, Berenson RA. Structuring payment for medical homes. Health Affairs 2010;29:852-8.

16. Rittenhouse DR, Shortell SM. The patient-centered medical home: Will it stand the test of health reform? JAMA 2009;301:2038-40.

17. Shay, K, Phibbs, C., Intrator, O, et al. Evaluating the costs for veterans receiving care in geriatric medical homes (GeriPACT). Innovations in Aging. 2017; Supplement 1:1329. 